\title{
Masses and mass distributions of protoplanetary disks
}

\author{
Lee Hartmann \\ E-mail: lhartm@umich.edu \\ Received 11 March 2008 \\ Accepted for publication 14 March 2008 \\ Published 16 July 2008 \\ Online at stacks.iop.org/PhysScr/T130/014012
}

Depatment of Astronomy, University of Michigan, 500 Church Street, Ann Arbor, MI 48109, USA

\begin{abstract}
I discuss some recent estimates of protoplanetary disk masses from dust emission, and suggest that masses have been systematically underestimated. I also consider the implications of recent results on the outbursting FU Ori objects, which demonstrate the need for substantial amounts of mass in disks within $1 \mathrm{AU}$, at least at early evolutionary stages. These considerations lend more weight to suggestions that protoplanetary disks have relatively massive 'dead zones', which may aid in the formation of the so-called 'transitional disk' systems, objects in which the inner disk has been strongly cleared of small dust even though the outer disk is very substantial.

PACS numbers: 97.82.--j, 97.82.Fs, 97.82.Jw
\end{abstract}

\section{Introduction}

Understanding initial protoplanetary disk masses is clearly important in developing a picture of planet formation. It is thought that most of the mass of a typical star is accreted through its disk (Hartmann et al 2006), suggesting that disks may be very massive in early evolutionary phases, and thus making planetary formation by gravitational fragmentation viable (see discussion by Boss 2008). In contrast, typical disk masses found from millimeter-wave and sub-millimeter-wave dust emission are much lower (e.g. Beckwith et al 1990). A very recent comprehensive survey of the Taurus star-forming region suggests a median disk mass of $5 \times 10^{-3} M_{\odot}$ (Andrews and Williams 2005), albeit with a large scatter. This median mass estimate is an order of magnitude lower than what is required for gravitational instability, though a few objects seem to be close to the instability limit.

However, disk masses estimated from dust emission are uncertain because we really do not understand the dust opacity. It is clear that the dust in protoplanetary disks has evolved considerably from its state in the interstellar medium; in particular, much larger dust particles are needed, with sizes of a millimeter or more, to account for the dependence of opacity on wavelength (Andrews and Williams 2005; Beckwith and Sargent 1991). Moreover, the dust opacity often assumed in analyzing may well be near the maximum plausible value.

Figure 1 shows opacity calculations from D'Alessio et al (2001) for power-law distributions of dust sizes for a typical expected dust composition. The dashed horizontal line in the upper panel shows the typical opacity used in estimating disk masses. Thus, these calculations indicate that the usual opacity is near the maximum obtainable with this set of assumptions. Moreover, the detection of a dust spectral index $\beta \sim 1$ (e.g. Andrews and Williams 2007) does not ensure a maximum size of particles; all that matters is the size distribution of the dust through the millimeter region. Thus, growth to maximum sizes of arbitrary magnitude is possible while maintaining a fixed spectral index. As shown in the middle panel of figure 1, a power-law distribution in size of $N(a) \propto a^{-3.5}$ yields an opacity spectral index $\beta \sim 1$ (horizontal dashed line in middle panel), a typical or median value inferred from observations (e.g. Andrews and Williams 2007). Obviously, there is much more parameter space at this value of $\beta$ at large dust sizes than the relatively narrow range which agrees with the usual millimeter opacity. Thus, dust growth is more likely to yield dust opacities lower than the typical adopted value than high dust opacities; in turn, this implies that disk masses are systematically underestimated.

In addition, a significant number of exoplanets have now been discovered which have $M \sin i$ larger than one Jupiter mass, sometimes having several Jupiter masses. This seems to be difficult to achieve with the median dust disk mass estimate, which is close to the so-called minimum mass solar nebula (MMSN), $\sim 10^{-2} M_{\odot}$ needed to form Jupiter and little more. It is difficult to believe that such systems can be formed without having an initial disk mass considerably larger 


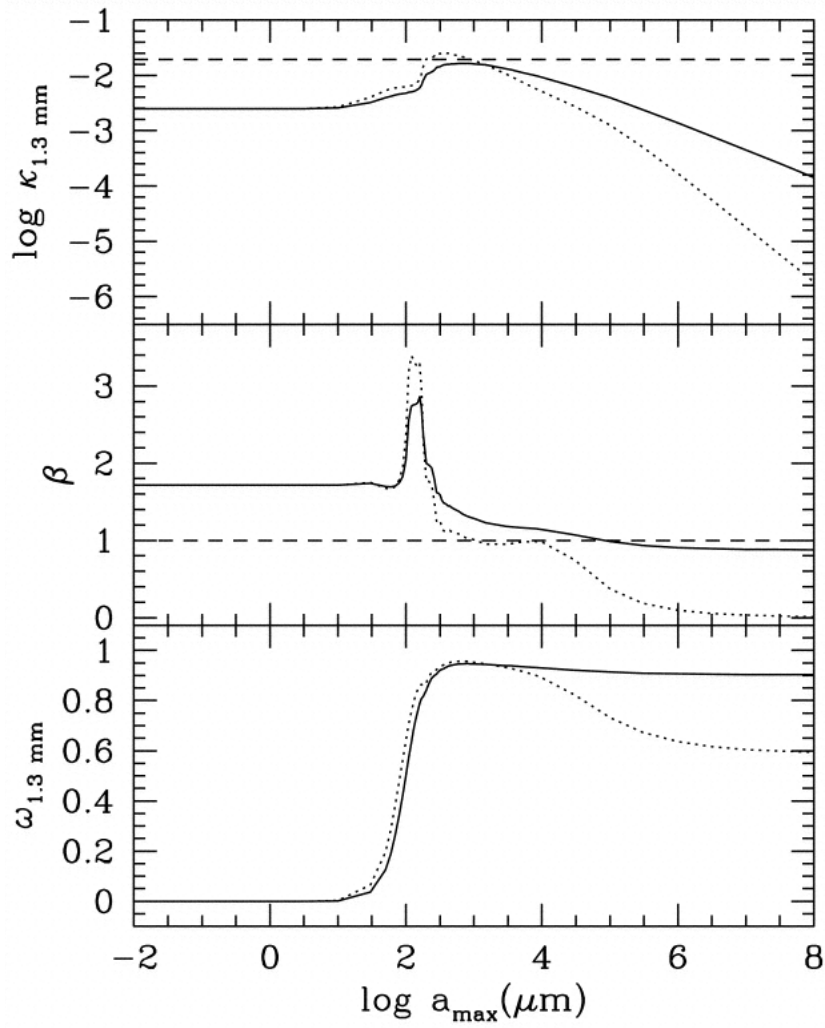

Figure 1. Optical properties for spherical dust grains of 'standard' composition, adopting a power-law distribution of radii

$N(a) \propto a^{-p g}$, with $p g=3.5$ (solid curves) and $p g=2.5$ (dotted curves), with a maximum grain radius $a_{\max }$. The upper panel shows the mass absorption coefficient at $\lambda=1.3 \mathrm{~mm}$ as a function of $a_{\max }$; the horizontal dashed line represents the frequently adopted opacity at $1.3 \mathrm{~mm}$. The middle panel shows the value of $\beta$ calculated between 0.769 and $1.3 \mathrm{~mm}$ for the same cases as the upper panel. The horizontal dashed line denotes $\beta=1$. The bottom panel shows the variation of the albedo at $1.3 \mathrm{~mm}$. From D'Alessio et al (2001).

than the MMSN, again suggesting that the current disk mass estimates are systematically low.

\section{Gas accretion and disk masses}

Hartmann et al (1998) pointed out that typical accretion rates for $\mathrm{T}$ Tauri stars place lower limits on initial disk masses. There are significant uncertainties in measuring accretion rates, and this constraint is only statistical in nature because the precise ages of individual $\mathrm{T}$ Tauri stars are uncertain, as is the timescale over which accretion decays are uncertain. Nevertheless, limits on disk masses from accretion are important because they measure gas, not dust.

Hartmann et al (1998) pointed out that typical estimates of classical $\mathrm{T}$ Tauri star (CTTS) accretion rates $\sim$ $10^{-8} M_{\odot} \mathrm{yr}^{-1}$ (figure 2 ) over lifetimes of $\sim 2 \mathrm{Myr}$ require minimum disk masses of $0.02 M_{\odot}$, somewhat larger than the median dust mass estimate of Andrews and Williams (2005). In addition, it is possible that the accretion rates have been underestimated systematically by not accounting for red-optical excess emission, perhaps by a factor of two or so (White and Hillenbrand 2004). Finally, such estimates are generally lower limits to disk masses, because, obviously, some mass is left behind. These considerations again suggest that typical dust mass determinations are underestimates.

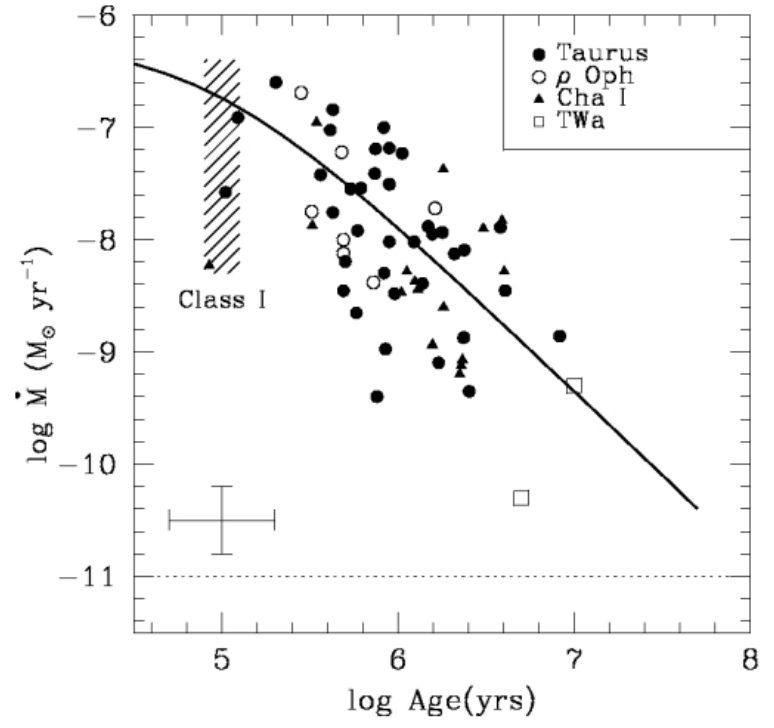

Figure 2. Mass accretion rates determined from blue-ultraviolet excess emission as a function of the estimated stellar age, for typical $\mathrm{T}$ Tauri stars in a variety of regions. The solid curve is not a fit to the data, but is a simple disk model calculation which assumes purely viscous accretion (Hartmann et al 1998). Courtesy N Calvet.

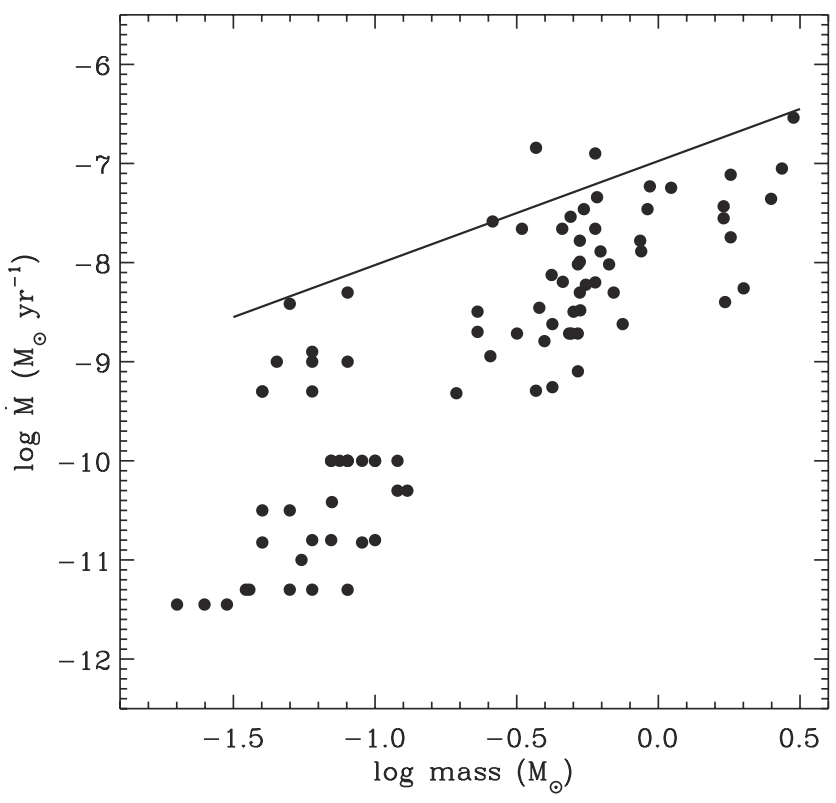

Figure 3. Mass accretion rates for young stars as a function of stellar mass. From Hartmann et al (2006).

As shown in figure 3, disk accretion rates have a strong dependence upon the mass of the central star. The solid line in figure 1 denotes the accretion rate as a function of mass at which 0.1 of the stellar mass would be accreted in $10^{6} \mathrm{yr}$. Accretion rates above this line, if sustained over $1 \mathrm{Myr}$, would result in accretion of more than $0.1 M_{*}$, suggesting that the initial $\mathrm{T}$ Tauri disk might have been gravitationally unstable. The steep dependence of accretion rate on mass results in the intermediate-mass $\left(\sim 1-2.5 M_{\odot}\right)$ classical $\mathrm{T}$ Tauri stars (Calvet et al 2004) lying much closer to this limiting line than the lower-mass objects. Given the obvious point that the accretion of these more massive objects to date has not exhausted their disk masses. Figure 1 suggests that gravitational instability is a real possibility for at least stars of masses $=1 M_{\odot}$. 
This is not to say that $\mathrm{T}$ Tauri disks must be gravitationally unstable, especially globally; nor does it automatically follow that gravitational instability is the favored mechanism for giant planet formation. One of the problems I have with gravitational instability for planet formation is the following: even if the fragments are initially of low mass, why will not they continue to accrete? As mentioned above, one might expect that disk self-gravity is most favorable at early times, when mass is still being added to the disk, and the disk may not be able to drain onto the central star fast enough. But in this case continued infall, not to say continued accretion from the outer disk, will tend to result in mass being accreted by the fragment(s). Bate and Bonnell (1997) found that even starting with a binary of a few Jupiter masses results in buildup to a binary star system, due to the increasing amount of matter accreted by both objects. Why this should not occur in the GI model is unclear.

\section{Dead zones and the FU Ori objects}

It is plausible that gravitational torques are a major mechanism for transferring most of the mass of the initial disk onto the central star. Eventually, as material drains out of the disk, one expects the disk to evolve to a marginally stable state in the absence of any other mechanism of angular momentum transfer. The magnetorotational instability (MRI; Balbus and Hawley 1998) is currently the favored mechanism for accretion in ionized disks. The question is: are protoplanetary disks sufficiently ionized for the MRI to operate?

Gammie (1996) pointed out that non-thermal ionization would be necessary to enable the MRI to operate in most regions of $\mathrm{T}$ Tauri disks. He originally suggested that cosmic rays were responsible for this ionization, although x-rays from the central star now seems more probable (e.g. Igea and Glassgold 1999). Gammie's insight was to suggest that accretion might proceed in upper disk layers, while central regions would remain non-turbulent or 'dead', except in the outermost regions where the ionizing radiation could completely penetrate the low-surface density regions, or in the innermost regions, where temperatures rise sufficiently that thermal ionization is important (figure 4).

For x-rays (or cosmic rays) to produce enough ionization to activate the MRI, it seems that substantial depletion of small dust is needed in the upper layers. Sano et al (2000) studied the effects of grain depletion and grain size on the surface density of the active layer, and estimated that $10^{-3}-10^{-4}$ were needed to produce sufficient depletion to activate reasonable amounts of disk matter. Some crude estimates of the possible settling of dust can be made from modeling recent Spitzer IRS spectra, suggesting depletions of factors of order $10^{-2}-10^{-3}$ (Furlan et al 2006). Some small grains must remain in the upper layers at several scale heights to explain both the overall flux levels and the observed silicate emission features (D'Alessio et al 2001). In addition, there is no evidence that stars with the most evidence for settling have larger mass accretion rates (Furlan et al 2006).

The layered disk model is affected by many aspects of non-thermal ionization (e.g. grain growth and settling, $\mathrm{x}$-ray

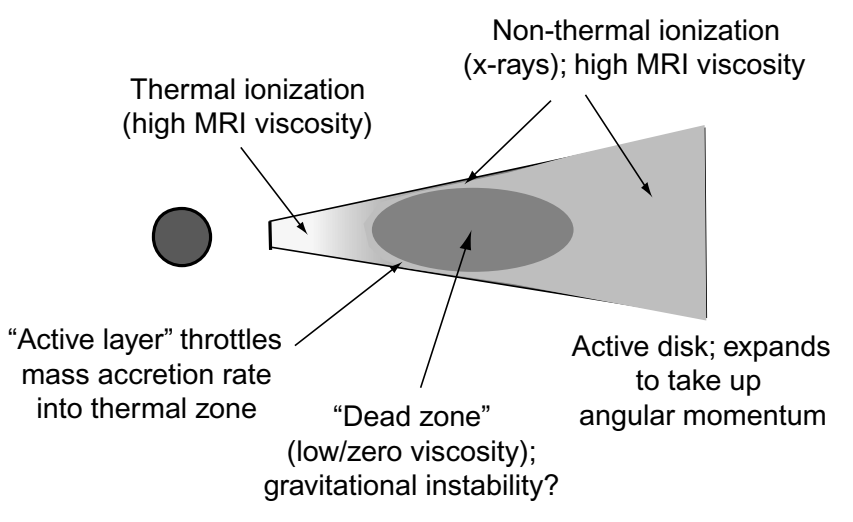

Figure 4. Schematic version of a 'layered' accretion disk. The innermost regions can be sufficiently ionized for the MRI to operate because the disk is warm, with estimated temperatures $=1000 \mathrm{~K}$; the outer regions may also be the site of an 'active' MRI due to non-thermal ionization by cosmic or x-rays. At intermediate radii, the disk is too cold for thermal ionization to be effective, and have too high a surface density for cosmic or x-rays to penetrate; this region may then be relatively inert, not subject to the MRI.

fluxes, presence or absence of cosmic rays) whose effects could range from allowing a full MRI to operate to shutting it off completely. The ubiquity of disk accretion among young stars suggests that some process might be operating which minimizes the possible variations in MRI activity. In the simple layered disk picture, the mass accretion rate is generally not a constant but decreases with radius Gammie 1996. This results in a pileup of matter over time in inner disk regions - which would be very difficult to detect with current interferometry, given size scales and also that these regions are likely to be very optically-thick. One might conjecture that, if the MRI is relatively inactive in a middle-radius region of the disk, material will then tend to pile up (fed from the outer, MRI-active disk; figure 4) and eventually could become gravitationally unstable, at which point mass transfer would begin to the inner disk.

Is there any evidence for such a disk structure? The answer is yes, the FU Ori objects (Hartmann and Kenyon 1996). These systems exhibit strong outbursts of accretion, $\sim 10^{-4} M_{\odot} \mathrm{yr}^{-1}$ for the order of a century, resulting in the accretion of order $0.01 M_{\odot}$ during a single outburst. This short timescale indicates that this significant amount of mass must reside within a modest radius - otherwise it cannot accrete onto the central star within the required (evolutionarily short) timescale.

Recent analysis of spectral energy distributions with the IRS instrument on board the Spitzer Space Telescope indicate that the accreting region of the FU Ori resides within a radius $\sim 0.5-1$ AU (Zhu et al 2007). For reference, one should note that the standard MMSN contains $\sim 0.002 M_{\odot}$ within $1 \mathrm{AU}$. Indeed, Zhu et al (2007) estimate that the disk must be close to gravitational instability at this radius. This result is consistent with the model proposed by Armitage et al (2001), who showed that large outbursts of accretion could be obtained by gravitational instabilities feeding an inner disk at scales of order an $\mathrm{AU}$, based on assuming the presence of a dead zone where the mass piles up. While the FU Ori objects probably represent an early stage of star/disk evolution (Hartmann and Kenyon 1996), it is still important to consider potential evidence for a dead zone at any age. 
The dead zone model suggests that the material might pile up in inner disk regions, even when infall is not present; and, speculatively, instabilities resulting from slow, less massive, unstable regions might result in the smaller 'Exor' outbursts (Herbig 1977), as evidenced in recent events (Acosta-Pulido et al 2007, Herbig 2007, Reipurth and Aspin 2004). In addition, Matsumura et al (2007) showed that the development of a dead zone might be important not only for understanding planet formation, but alsoplanet migration as well.

Another aspect of a dead zone model is this: the presence of a massive, dense region might be more favorable for the formation of planetesimals. In this regard, the properties of the so-called 'transitional disks' (Calvet et al 2005, D'Alessio et al 2005) are particularly interesting. The transitional disks typically show substantial mid-to-far-infrared emission, suggesting an outer, massive disk, but with an inner region depleted of small dust, and in some cases reduced gas accretion. These disks do not show gaps, as would be expected for the formation of a single giant planet, but inner disk holes (relatively evacuated) over a large range of radii. This seems to suggest nearly simultaneous evolution over a significant range of radii, which is unusual in that evolutionary timescales tend to increase with increasing radius, assuming a surface density which declines with increasing radius no faster than $r^{-3 / 2}$ (Chiang and Goldreich 1997, equation (15)). A dead zone model tends to have high densities over a significant range of radial distances, suggesting that this would be the ideal situation to 'evacuate' the inner disk at nearly a single epoch. Further studies on disk structure inferred from spectral energy distributions and other techniques will help test the idea that dead zones lead to approximately simultaneous giant planet formation over decades in radius.

\section{Acknowledgment}

I thank the organizers of this stimulating conference for the opportunity given to attend.

\section{References}

Acosta-Pulido J A et al 2007 Astron. J. 133 2020-36

Andrews S M and Williams J P 2005 Astrophys. J. 631 1134-60

Andrews S M and Williams J P 2007 Astrophys. J. 659 $705-28$

Armitage P J, Livio M and Pringle J E 2001 Mon. Not. R. Astron. Soc. 324 705-11

Balbus S A and Hawley J F 1998 Rev. Mod. Phys. 70 1-53

Bate M R and Bonnell I A 1997 Mon. Not. R. Astron. Soc. 285 33-48

Beckwith S V W and Sargent A I 1991 Astrophys. J. 381 250-8

Beckwith S V W, Sargent A I, Chini R S and Guesten R 1990 Astron. J. 99 924-45

Boss A P 2008 Phys. Scr. T130 014020

Calvet N 2005 Astrophys. J. 630 L185-8

Calvet N, Muzerolle J, Briceño C, Hernández J, Hartmann L, Saucedo J L and Gordon K D 2004 Astron. J. 128 1294-318

Chiang E I and Goldreich P 1997 Astrophys. J. 490 368-76

D'Alessio P, Calvet N and Hartmann L 2001 Astrophys. J. 553 321-34

D'Alessio P et al 2005 Astrophys. J. 621 461-72

Furlan E et al 2006 Astrophys. J. Suppl 165 568-605

Gammie C F 1996 Astrophys. J. 457355

Hartmann L, Calvet N, Gullbring E and D'Alessio P 1998 Astrophys. J. 495385

Hartmann L, D'Alessio P, Calvet N and Muzerolle J 2006 Astrophys. J. 648 484-90

Hartmann L and Kenyon S J 1996 Annu. Rev. Astron. Astrophys. 34 207-40

Herbig G H 1977 Astrophys. J. 217 693-715

Herbig G H 2007 Astron. J. 133 2679-83

Igea J and Glassgold A E 1999 Astrophys. J. 518 848-58

Matsumura S, Pudritz R E and Thommes E W 2007 Astrophys. J. $6601609-23$

Reipurth B and Aspin C 2004 Astrophys. J. 606 L119-22

Sano T, Miyama S M, Umebayashi T and Nakano T 2000 Astrophys. J. $\mathbf{5 4 3} 486-501$

White R J and Hillenbrand L A 2004 Astrophys. J. 616 998-1032

Zhu Z, Hartmann L, Calvet N, Hernandez J, Muzerolle J and Tannirkulam A K 2007 Preprint 707 